\title{
An experimental model for the measurement of renal function after temporary ureteral obstruction in the rat
}

\author{
S. Tucci Jr., \\ M.C. Beduschi, \\ S.A. Mazzetto \\ and J.C. Vanni
}

\author{
Laboratório de Cirurgia Experimental, Departamento de Cirurgia, \\ Ortopedia e Traumatologia, Faculdade de Medicina de Ribeirão Preto, \\ Universidade de São Paulo, 14049-900 Ribeirão Preto, SP, Brasil
}

\section{Correspondence \\ S. Tucci Jr. \\ Departamento de Cirurgia, \\ Ortopedia e Traumatologia FMRP, USP \\ 14049-900 Ribeirão Preto, SP Brasil \\ Fax: 55 (016) 633-0836}

Research supported by FAEPA. Publication supported by FAPESP. .....................

Received July 11, 1996 Accepted May 19, 1997

\begin{abstract}
We present the results obtained with a ureterovesical implant after ipsilateral ureteral obstruction in the rat, suitable for the study of renal function after deobstruction in these animals. Thirty-seven male Wistar rats weighing 260 to $300 \mathrm{~g}$ were submitted to distal right ureteral ligation and divided into 3 groups, $\mathrm{A}(\mathrm{N}=13,1$ week of obstruction), $\mathrm{B}(\mathrm{N}=14,2$ weeks of obstruction) and $\mathrm{C}(\mathrm{N}=10,3$ weeks of obstruction). The animals were then submitted to ureterovesical implantation on the right side and nephrectomy on the left side. During the 4-week follow-up period serum levels of urea and creatinine were measured on the 2nd, 7th, 14th, 21st and 28th day and compared with preoperative levels. The ureterovesical implantation included a psoas hitch procedure and the ureter was pulled into the bladder using a transvesical suture. During the first week of the postoperative period 8 animals died, 4/13 in group A (1 week of obstruction) and 4/14 in group B (2 weeks of obstruction). When compared to preoperative serum levels, urea and creatinine showed a significant increase $(\mathrm{P}<0.05)$ on the 2nd postoperative day in groups $\mathrm{A}$ and $\mathrm{B}$, with a gradual return to lower levels. However, the values in group B animals were higher than those in group A at the end of the follow-up. In group C, 2/10 animals (after 3 weeks of obstruction) were sacrificed at the time of ureterovesical implantation due to infection of the obstructed kidneys. The remaining animals in this group were operated upon but all of them died during the first week of follow-up due to renal failure. This technique of ureterovesical implantation in the rat provides effective drainage of the upper urinary tract, permitting the development of an experimental model for the study of long-term renal function after a period of ureteral obstruction.
\end{abstract}

\section{Introduction}

Urinary obstruction, together with infectious processes, continues to be the most frequent consequence of diseases that affect the urinary tract, potentially causing renal parenchymal damage of an irreversible na-
Key words

- Kidney function

- Rat

- Ureteral obstruction 
removed at the time of obstruction release (1). In contrast, recovery of renal function was demonstrated in humans after 56 and 69 days of total ureteral obstruction $(2,3)$.

The demonstration of recovery of renal function after ureteral obstruction is a difficult task and needs to be thoroughly documented for credibility. Dogs are frequently employed among experimental animals but their use has the disadvantage of requiring sterilized instruments and a more refined anesthetic technique, in addition to more cumbersome pre- and postoperative procedures. The rat, in contrast, is easier to handle during the pre- and postoperative periods, requires a simple anesthetic technique and the need for asepsis is not absolute. In addition, several animals can be operated upon within a shorter period of time, resulting in much lower costs.

We present here a technique for ureterovesical implantation in the rat after ipsilateral ureteral obstruction and the results obtained with it, which will permit the development of a model for the study of long-term renal function after deobstruction in these animals.

\section{Material and Methods}

The study was conducted on 37 male Wistar rats weighing 260 to $300 \mathrm{~g}$, supplied by the Central Animal House of the Faculty of Medicine of Ribeirão Preto, University of São Paulo. The animals were submitted to distal ureteral ligation on the right side and divided into 3 groups. Group A $(\mathrm{N}=13)$ was submitted to 1 week of ureteral obstruction, group $\mathrm{B}(\mathrm{N}=14)$ to 2 weeks and group $\mathrm{C}(\mathrm{N}$ $=10)$ to 3 weeks of obstruction.

The animals were reoperated upon at the appropriate time and submitted to a ureterovesical implant on the right side. On this occasion, blood was collected for measurement of basal serum urea and creatinine. Nephrectomy of the non-obstructed kidney was then performed, so that the animal was left only with the previously obstructed kidney, whose ureter was implanted into the bladder.

During the postoperative period the animals were placed in cages with free access to ration and water. Blood samples for the determination of serum urea and creatinine were obtained on the $2 \mathrm{nd}, 7 \mathrm{th}, 14 \mathrm{th}, 21 \mathrm{st}$ and 28th day after ureteral implantation, when the animals were sacrificed.

\section{Description of the techniques}

All surgical procedures were performed under inhalatory ethyl ether anesthesia.

Ureteral obstruction. We chose the right ureter for distal ligation because this region is more easily exposed than the left due to the presence of the loops of the large bowel. The abdominal area was shaved and the animals were submitted to median laparotomy of approximately $2 \mathrm{~cm}$ in length in the more caudal region of the abdomen. The juxtavesical ureter was identified and dissected with a thin curved forceps for a distance only sufficient for the passage of a 5-0 Vicryl ${ }^{\mathrm{TM}}$ suture which was used for ligation, followed by section of the distal ureteral wall to ensure complete obstruction. The laparotomy incision was closed on a single plane with 4-0 Prolene ${ }^{\mathrm{TM}}$ sutures.

Ureteral implant. The abdomen was again shaved and a xipho-pubic median laparotomy was performed. The intestinal loops were positioned on the left outside the abdominal cavity and protected with gauze soaked in $0.9 \%$ saline, so as to obtain an ample operating field. The ureter was easily recognized on the basis of its caliber and was carefully dissected and freed from the parietal peritoneum.

A small extension of the psoas muscle was then exposed close to the site of the original ureteral ligation and care was taken to verify that the vesical dome easily reached the exposed region of the psoas muscle. The dome was then fixed to the psoas with two 5$0 \mathrm{Vicryl}^{\mathrm{TM}}$ stitches separated by a distance 
of approximately $2 \mathrm{~mm}$. These stitches passed through all the layers of the vesical wall. Using thin scissors, the vesical wall was extensively opened anterior to the midline, with exposure of its lumen from the region close to the vesical neck to the stitches for fixation to the psoas.

Using 5-0 or 6-0 Vicryl ${ }^{\mathrm{TM}}$ suture, a stitch was taken transfixing the posterior wall of the bladder from the outside in along the midline and close to the vesical neck, and exteriorized through the incision in the anterior wall. The proximal ureteral end was identified, with care taken not to leave the ureter twisted around its axis. Using the same Vicryl ${ }^{\mathrm{TM}}$ suture exteriorized through the vesical incision, a stitch was taken inside the lumen of the ureter transfixing the posterior ureteral wall at a distance of 1 to $2 \mathrm{~mm}$ from the sectioned ureteral end. The posterior vesical wall was then transfixed again from the inside out, with the needle exiting close to the initial site of entry (Figure 1, left). In summary, an inverted "U" stitch was taken with the suture entering through the posterior vesical wall, transfixing the ureter and exiting close to the site of entry. When the knot was tied on the outer surface of the posterior vesical wall, the ureter was gently stretched, positioned inside the bladder and fixed close to the vesical neck. This knot was tied delicately, being barely sufficient to permit the juxtaposition of the ureter to the vesical wall. The anterior vesical wall was then sutured with continuous 5-0 Vicryl ${ }^{\mathrm{TM}}$ starting from the angle close to the vesical neck and ending close to the site of entry of the ureter into the bladder (Figure 1, right). Careful suturing is needed at this site in order not to constrict the ureteral lumen. The abdominal wall was closed on two planes with continuous 4-0 Prolene ${ }^{\mathrm{TM}}$ sutures.

Left nephrectomy. The procedure was performed by exposing, ligating and sectioning the renal peduncle including the artery, renal vein and ureter.

Blood samples. Samples for the determi-
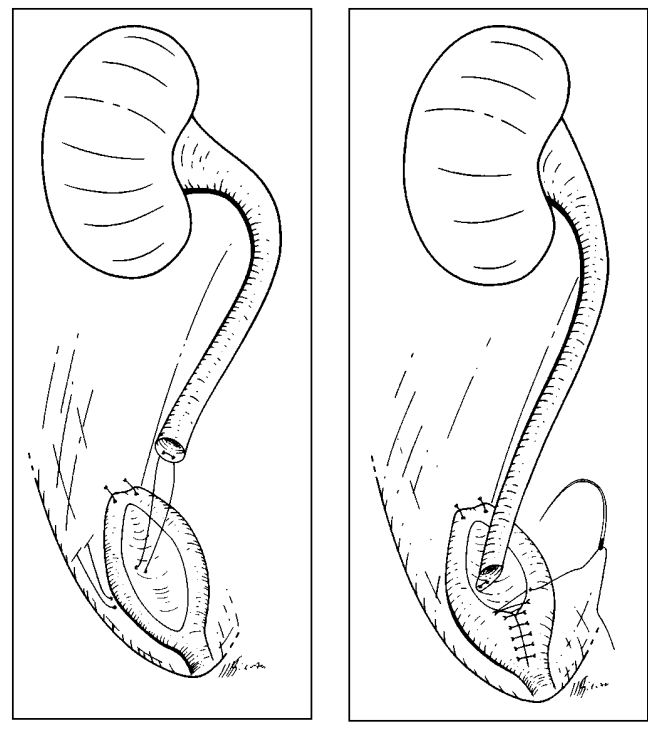

Figure 1 - Ureteroneocystostomy in the rat. Left, Bladder dome fixed to psoas muscle and inverted " $U$ " stitch transfixing bladder and ureteral walls. Right, Ureter positioned inside the bladder, fixed to the posterior bladder wall.

nation of basal serum urea and creatinine were collected by puncture of the vena cava immediately before nephrectomy of the nonobstructed kidney. During the postoperative period, blood samples were obtained by successive tail section.

Data from serum urea and creatinine determinations were analyzed statistically by the Mann-Whitney test.

\section{Results}

Ureteral ligation was effective in promoting obstruction, resulting in ureterohydronephrosis. The ureter reached an outer diameter of 1 to $3 \mathrm{~mm}$.

During the first week after ureteral implantation 4/13 animals (30.7\%) in group A died, 2 of them of peritonitis with urine in the abdominal cavity. It was not possible to identify the cause of death for the other two. Four/14 (28.5\%) deaths occurred in group B, 1 of them secondary to ureteral obstruction by the vesical suture and 3 of them due to peritonitis. In 2 of these animals there was urine in the abdominal cavity, due to a ureteral fistula in one. It was not possible to identify the site of origin of the urine present in the abdomen for the remaining case. In the last animal, peritonitis was caused by exten- 
Figure 2 - Effect of right ureteroneocystostomy and contralateral nephrectomy following one or two weeks of right ureteral obstruction on serum urea (top) and creatinine (bottom). Data are reported as means \pm SEM, as $\mathrm{mg} \%$ for 6 rats in each group. ${ }^{*} \mathrm{P}<0.05$ compared to day 0 (Mann-Whitney test).
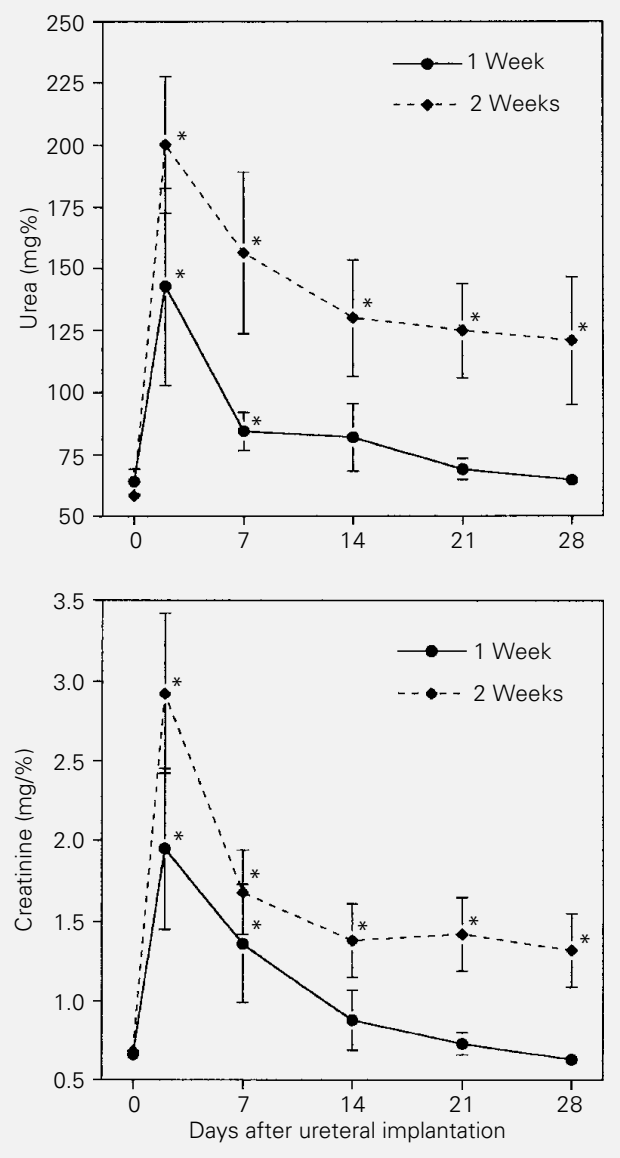

sion of the infection in the suture of the abdominal wall. All other animals in these groups, 9/13 after one week of obstruction and 10/14 after 2 weeks of obstruction, survived without complications until the end of the observation period.

After 3 weeks of obstruction, 2 animals of group $\mathrm{C}$ were sacrificed at the time of ureterovesical implantation due to infection of the obstructed kidneys. The remaining 8 animals in this group were operated upon but all of them died during the first week of follow-up due to renal failure, confirmed by very high levels of serum urea and creatinine. Five of these animals were inspected after death and no obstruction was found at the site of ureterovesical implantation.

Inspection of the urinary tract of groups $A$ and $B$ after sacrifice revealed a reduction of the ureteral caliber in all animals com- pared to that observed at the time of implantation. The vesical suture presented good healing, and no abscesses or fluid collections were observed at the site of implantation.

Serum urea and creatinine were determined in 6 animals from group A and in 6 from group B. When compared to preoperative levels, there was a significant increase in these values on the 2 nd postoperative day in both groups $(\mathrm{P}<0.05)$, with a gradual return to lower values during the observation period of 4 weeks (Figure 2). In group A these values were already similar to the preoperative ones during the $3 \mathrm{rd}$ week, a fact that was not observed in group B.

At the end of the 4th week of follow-up the pre- and postoperative levels of urea and creatinine showed a significant difference in animals of group $\mathrm{B}(\mathrm{P}<0.05)$ and a nonsignificant difference in group $\mathrm{A}(\mathrm{P}>0.05)$.

\section{Discussion}

Ureterovesical implants in small experimental animals such as rabbits and rats have been mainly employed for renal transplant. However, since the normal ureter of the rat is of extremely reduced caliber and subject to complications such as stenosis and fistulae in the anastomosis region, this implant is more frequently performed by suturing the bladder segment that surrounds the donor's ureter to the bladder of the receptor, with uretero-ureteral anastomosis being limited to cases of orthotopic renal transplant (4-9).

The technique described here was developed in order to permit reconstruction of the rat urinary tract after a period of ureteral obstruction. Some details of the technique deserve discussion: a) the rat ureter is an extremely delicate structure. Thus, before ligation, the ureter should be dissected along the shortest possible distance, reducing the formation of local adhesions. This facilitates later dissection for implantation and permits to obtain a ureter with more viable walls. 
Furthermore, since the ureter is closely related to the gonadal vessels, more extensive dissection may provoke damage and local bleeding requiring ligation of the vessels. $b$ ) The use of two 5-0 Vicryl ${ }^{\mathrm{TM}}$ stitches separated by a distance of $2 \mathrm{~mm}$ insures better vesical fixation while at the same time maintaining the anterior wall exposed for incision. In the first animals in the series we used only one stitch for fixation of the bladder. If dehiscence of this stitch should occur, the bladder will move from its position, possibly separating from the ureter. c) The passage of the transfixing stitch through the ureteral wall is facilitated by the increased caliber of the ureter, with no need for optical magnification. The knot on the outer surface of the vesical wall only serves to maintain the ureter in its new position during the healing phase and should be tied delicately, without tension, which may lead to tissue necrosis and ineffective healing. d) During implant it is important that the intestinal loops that were positioned outside the abdominal cavity are covered with gauze moistened with $0.9 \%$ saline. This prevents drying, reducing the fluid loss presented by the animal during this period. Furthermore, when the incision is closed, the instillation of approximately $10 \mathrm{ml} 0.9 \%$ saline into the peritoneal cavity is usually sufficient for animal repletion and maintenance during the immediate postoperative period. e) Monofilament 4-0 suture with closure on two planes is more indicated for abdominal wall suturing, the use of the former being important for the muscles and peritoneum and the use of the latter being important for the skin. This procedure provides more safety against the occurrence of dehiscence of this suture, reducing the incidence of infectious processes observed in the abdominal wall of the rat when multifilament sutures are used. It should be remembered that the animal will probably go through a period of renal insufficiency during which it will be more subject to infections and the healing process may be deficient.
The anesthetic technique used, ether inhalation, has the disadvantage of inducing bronchial secretion and respiratory depression, possibly causing the death of the animal. Thus, when using this technique the surgeon should periodically check respiratory rate and tissue perfusion. The advantage of the technique is that it is inexpensive and permits the animal to recover its normal activity a few minutes after the end of anesthesia.

Serum urea and creatinine levels measured during the postoperative period were significantly increased on the 2nd day in groups A and B when compared to preoperative levels, reflecting the reduction in renal function due to the period of urinary obstruction. Since the animals were left only with the previously obstructed kidney, the gradual reduction of these values was compatible with improved renal function, leading us to conclude that the ureterovesical implant was effective in permitting sufficient drainage of the upper urinary tract.

The observation that the serum urea and creatinine levels of group B did not return to basal values during the postoperative follow-up of 4 weeks suggests the need for a longer time after deobstruction to reach normal levels due to a more extensive lesion of the renal parenchyma secondary to the longer period of ureteral obstruction. However, another possibility to be considered is the presence of some degree of ureteral obstruction caused by cicatrization of the bladder. In the only animal in which severe obstruction occurred there was a marked increase in hydronephrosis followed by death. Evaluation after sacrifice of all surviving animals showed a reduction of ureterohydronephrosis. On this basis, we emphasize that suture of the anterior vesical wall should be performed with great care.

We believe that the deaths of rats in group $\mathrm{C}$ were due to a severe lesion of the renal parenchyma because there was no obstruction of the ureter as observed during 
postmortem evaluation of 5 animals. As observed in dogs (1), the rat also does not survive ureteral obstruction of 3-week duration when the contralateral kidney is removed at the time of obstruction release. This is a limitation of the model.

The deaths observed during the early postoperative period were mostly due to technical errors. Five animals presented peritonitis, with urine in the abdominal cavity. The ureteral fistula was either due to manipulation of the ureter with an inappropriate forceps or was secondary to ischemia of the wall caused by the extensive dissection for implantation. In the other rats it was impossible to determine the site of urine leakage into the abdomen but the most probable cause of this event should be a leak in the vesical suture.

In one animal there was ureteral stenosis at the site of entry into the bladder, secondary to excessive closure of the vesical wall, and in another peritonitis started due to infection of the abdominal wall. Improvement of the surgical technique, therefore, should reduce the number of deaths in future series.

The present technique of ureterovesical implantation in the rat provided effective drainage of the upper urinary tract, with $70 \%$ survival for 1-2 weeks, permitting the development of an experimental model of measurement of the renal function after a period of ureteral obstruction.

\section{References}

1. Gillenwatter JY (1992). The pathophysiology of urinary tract obstruction. In: Walsh PC, Retik AB, Stamey TA \& Vaughan Jr ED (Editors), Campbell's Urology. 6th edn. W.B. Saunders, Philadelphia, 497-532.

2. Graham JB (1962). Recovery of kidney after ureteral obstruction. JAMA, 181: 993-995

3. Lewis HY \& Pierce JM (1962). Return of function after relief of complete ureteral obstruction of 69 days' duration. Journal of Urology, 88: 337-339.
4. Fisher B \& Lee S (1965). Microvascular surgical techniques in research, with special reference to renal transplantation in the rat. Surgery, 58: 904-914.

5. Lee $S$ (1967). An improved technique of renal transplantation in the rat. Surgery, 61: 771-773.

6. Dunn DC (1976). Orthotopic renal transplantation in the rabbit. Transplantation, 22: 427-433

7. Jacobsen IA (1978). Renal transplantation in the rabbit: a model for preservation studies. Laboratory Animals, 12: 63-70.
8. Silber SJ (1979). Microsurgery for laboratory experimentation. In: Silber SJ (Editor), Microsurgery. Williams \& Wilkins, Baltimore, 31-91.

9. Asfar SK, Catto GR \& Engeset J (1988). The rat renal transplant model. Details of microsurgical technique and complications. Journal of the Royal College of Surgeons of Edinburgh, 33: 314-317. 